\title{
Projeto de Controle Robusto Chaveado para Sistemas Lineares Incertos
}

\author{
Alvaro Coelho Gonçalves*, Wallysonn Alves de Souza* \\ * Coordenação de Ciências Matemáticas e Naturais, IFTO - Instituto \\ Federal de Educação, Ciência e Tecnologia do Tocantis, TO, \\ (e-mail: alvarosilna@gmail.com,wallysonn.souza@ifto.edu.br).
}

\begin{abstract}
The present work presents the analysis and switched robust control design for time invariant linear systems. The methodology presented is based on Linear Matrix Inequalities (LMIs) and uses a single quadratic Lyapunov function to design the feedback controller gains. The proposed control selects a single gain, of a set of previously designed gains, in a way that minimizes the time derivative of the Lyapunov function. The proposed methodology, even maintaining the conservatism of the existing ones in the literature, proved to be efficient in numerical simulations. This has been proven in the design of a control system with polytopic uncertainties for a magnetic levitator.

Resumo: O presente trabalho apresenta a análise e projeto de controle robusto chaveado para sistemas lineares invariantes no tempo. A metodologia apresentada é baseados em Desigualdades Matriciais Lineares (LMIs) e utiliza uma única função quadrática de Lyapunov para projetar os ganhos de realimentação do controlador. O controle proposto seleciona um único ganho, de um conjunto de ganhos previamente projetados, de forma que minimiza a derivada temporal da função de Lyapunov. A metodologia proposta, mesmo mantendo o conservadorismo das existentes na literatura, mostrou-se eficiente nas simulações numéricas. O que foi comprovado no projeto de um sistema de controle com incertezas politópicas para um levitador magnético.
\end{abstract}

Keywords: Switching controller; linear matrix inequalities (LMIs); polytopic uncertainties; robust control; magnetic levitator.

Palavras-chaves: Controlador chaveado; desigualdades matriciais lineares (LMIs); incertezas politópicas; controle robusto; levitador magnético.

\section{INTRODUÇÃO}

Com o desenvolvimento de técnicas ligadas a teoria de Lyapunov para a análise e síntese de controladores, baseados em Desigualdade Matriciais Lineares (LMIs), o final do século XX e o início deste século, foi marcado por um grande número descobertas sobre novas condições que garantem a estabilidade de sistemas lineares e não lineares. Além disso, devido ao grande número de problemas de caráter prático e acadêmico, tem crescido muito o interesse no estudo de sistemas chaveados Wicks et al. (1994); Liberzon and Morse (1999); Decarlo et al. (2000); Hespanha and Morse (2002); Geromel and Colaneri (2006); Souza et al. (2013, 2014); de Oliveira et al. (2014), principalmente no que diz respeito à análise de estabilidade e projeto de novos controladores, visto que, de uma forma geral, a resposta transitória de sistemas lineares invariantes no tempo pode ser melhorada ao utilizar controladores chaveados.

Recentemente, a comunidade científica tem demonstrado atenção para o estudo da análise de estabilidade e projeto de novos controladores, baseados em LMIs, com o chaveamento dos ganhos sobre a mesma planta, como pode ser visto em Silva et al. (2012) e Souza et al. (2013).

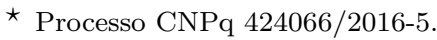

Em Souza et al. (2013), por exemplo, foi proposta uma nova metodologia de projeto de controle chaveado para uma classe de sistemas lineares com incertezas politópicas, que faz uso de uma única função de Lyapunov para projetar vários ganhos do controlador baseado em LMIs. Porém, esta metodologia de projeto de controle é limitada, visto que exige que a matriz $B$, matriz de controle do sistema, seja constante. Neste mesmo trabalho, a saída proposta, para o caso em que $B$ não é constante, foi aumentar a dimensão do sistema, por meio da inserção de um integrador, ou a garantia da estabilidade uniformemente "ultimate bounded", sob determinadas condições da matriz $B$.

Este trabalho tem como objetivo generalizar Souza et al. (2013) propondo uma metodologia de projeto de controladores robustos chaveados. A metodologia adotada garante a estabilidade assintótica global de sistemas lineares, com incertezas politópicas, e o controlador proposto seleciona um único ganho, que pertence a um conjunto de ganhos previamente projetados, é selecionado por uma lei de chaveamento que retorna o menor valor da derivada temporal da função de Lyapunov e, além disso, as LMIs propostas são igauis, em termos de conservadorismo, às estabelicidas em Souza et al. (2013) que, por sua vez, já são mais relaxadas do que as clássicas. Para comprovar a eficiência da técnica, foi projetado um sistema de controle para o levitador magnético e realizadas simulações computacio- 
nais. As simulações computacionais foram executadas no software Matlab, utilizando programação convexa na linguagem do YALMIP Lofberg (2004) com o solver SeduMi Sturm (1999).

Por conveniência, serão estabelecidas algumas notações que serão utilizadas neste trabalho:

$$
\begin{gathered}
\mathbb{K}_{r}=\{1,2, \ldots, r\}, r \in \mathbb{N} ; \quad x(t)=x ; \quad V(x(t))=V ; \\
(A, B, C, K)(\alpha)=\sum_{i=1}^{r} \alpha_{i}\left(A_{i}, B_{i}, C_{i}, K_{i}\right), \quad \alpha_{i} \geq 0 \\
\text { e } \sum_{i=1}^{r} \alpha_{i}=1, \alpha=\left[\alpha_{1}, \alpha_{2}, \ldots, \alpha_{r}\right]^{T},
\end{gathered}
$$

sendo $r=2^{s}$ e $s$ é o número de parâmetros e/ou funções incertas distintas nas matrizes.

\section{RESULTADOS PRELIMINARES}

Esta seção traz um estudo do controle de sistemas lineares, baseado em LMI's, seguindo a linha de Boyd et al. (1994).

\subsection{Estabilidade de Sistemas Lineares Realimentados}

Considere o sistema linear realimentado

$$
\dot{x}(t)=A x(t)+B u
$$

sendo $x \in \mathbb{R}^{n}$ o vetor de estado, $A \in \mathbb{R}^{n \times n}, B \in \mathbb{R}^{n \times m} \mathrm{e}$ $u \in \mathbb{R}^{m}$ a entrada de controle.

Suponha que todas as variáveis de estado estejam disponíveis para realimentação, uma lei de controle largamente usada na literatura Boyd et al. (1994), é

$$
u=-K x(t), \quad K \in \mathbb{R}^{m \times n}
$$

Substituindo (3) em (2), tem-se

$$
\dot{x}(t)=A x(t)-B K x(t)=(A-B K) x(t)
$$

Para garantir que o sistema linear realimentado (4) é estável segue o seguinte teorema.

Teorema 1. O sistema linear (4) é quadraticamente estabilizável se, e somente se, existir uma matrize simétrica definida positiva $X \in \mathbb{R}^{n \times n}$ e $M \in \mathbb{R}^{m \times n}$, tal que

$$
X A^{T}+A X-B M-M^{T} B^{T} \prec 0
$$

Se (5) é factível, o ganho do controlador é dado por $K=M X^{-1}$.

A demonstração do Teorema 1 pode ser vista, por exemplo, em Boyd et al. (1994).

O Teorema 1 garante a estabilidade do sistema realimentado (4), isto é, garantir que o sistema linear realimentado (4) seja estável é equivalente a encontrar a matriz $P$ de Lyapunov e o ganho estabilizante $K$.

\subsection{Estabilidade de Sistemas Lineares com Incertezas Politópicas}

Seja $A$ uma matriz que não seja precisamente conhecida. Define-se politopo de incertezas de $\mathcal{A}$ como sendo a combinação convexa de seus vértices, sendo considerado a combinação dos valores máximos e mínimos de cada valor e/ou parâmetro incerto. Assim, o politopo de incertezas tem $r=2^{s}$ vértices, sendo $s$ o número de componentes incertas distintas da matriz $A$. Matematicamente, pode-se escrever o politopo convexo da seguinte forma Leite et al. (2004):

$$
\begin{aligned}
\mathcal{A}=\left\{A(\alpha) \in \mathbb{R}^{n \times n}: A(\alpha)=\sum_{i=1}^{r} \alpha_{i} A_{i}\right. \\
\left.\operatorname{com} \alpha_{i} \geq 0, \quad \sum_{i=1}^{r} \alpha_{i}=1 \text { e } i \in \mathbb{K}_{r}\right\}
\end{aligned}
$$

Agora, considerando que o sistema (2) tenha incertezas politópicas, ele pode ser escrito como a seguir:

$$
\dot{x}=A(\alpha) x+B(\alpha) u=\sum_{i=1}^{r} \alpha_{i}\left(A_{i} x+B_{i} u\right)
$$

Sendo $x(t) \in \mathbb{R}^{n}$ o vetor de estado, $u(t) \in \mathbb{R}^{m}$ a entrada do controle, $A(\alpha), B(\alpha) \in \mathcal{A}$, matrizes que representam a dinâmica do sistema, com $A_{i} \in \mathbb{R}^{n \times n}, B_{i} \in \mathbb{R}^{n \times m}, i \in \mathbb{K}_{r}$.

Levando em consideração que todas as variáveis de estado estejam apropriadas para a realimentação, a lei de controle para realimentação de sistemas incertos será dada por (3). Substituindo (3) em (7), obtêm-se o sistema realimentado.

$$
\dot{x}=A(\alpha) x-B(\alpha) K x=\sum_{i=1}^{r} \alpha_{i}\left(A_{i}-B_{i} K\right) x
$$

Teorema 2. O ponto de equilíbrio $x=0$ do sistema linear com incertezas politópicas (8) é globalmente assintoticamente estável, se existem uma matriz simétrica definida positiva $X$ e uma matriz $M \in \mathbb{R}^{m \times n}$ tais que para todo $i \in \mathbb{K}_{r}$,

$$
X A_{i}^{T}+A_{i} X-B_{i} M-M^{T} B_{i}^{T} \prec 0
$$

Se as LMIs (9) são factíveis, para todo $i \in \mathbb{K}_{r}$, o ganho do controlador é dado por $K=M X^{-1}$.

Prova. Considere uma candidata a função de Lyapunov quadrática $V=x^{T} P x$. Portanto, de (8) tem-se que

$$
\begin{aligned}
\dot{V} & =\dot{x}^{T} P x+x^{T} P \dot{x} \\
& =\left[\sum_{i=1}^{r} \alpha_{i}\left(A_{i}-B_{i} K\right) x\right]^{T} P X+x^{T} P\left[\sum_{i=1}^{r} \alpha_{i}\left(A_{i}-B_{i} K\right) x\right] \\
& =\sum_{i=1}^{r} \alpha_{i} x^{T}\left(A_{i}-B_{i} K\right)^{T} P x+x^{T} P \sum_{i=1}^{r} \alpha_{i}\left(A_{i}-B_{i} K\right) x \\
& =x^{T}\left[\sum_{i=1}^{r} \alpha_{i}\left(A_{i}-B_{i} K\right)^{T} P+P \sum_{i=1}^{r} \alpha_{i}\left(A_{i}-B_{i} K\right)\right] x \\
& =x^{T}\left[\sum_{i=1}^{r} \alpha_{i}\left(A_{i}^{T} P-K^{T} B_{i}^{T} P+P A_{i}-P B_{i} K\right)\right] x
\end{aligned}
$$

Como $\alpha_{i} \geq 0, i \in \mathbb{K}_{r}$ e $\sum_{i=1}^{1} \alpha_{i}=1$, então em (10), $\dot{V}<0, \quad \forall x \neq 0$, se

$$
A_{i}^{T} P-K^{T} B_{i}^{T} P+P A_{i}-P B_{i} K \prec 0
$$

Definindo $X=P^{-1}$ e $M=K X$, multiplica-se as desigualdades (11) pela direita e pela esquerda por $X$, obtendo-se:

$$
X A_{i}^{T}+A_{i} X-B_{i} M-M^{T} B_{i}^{T} \prec 0 .
$$

$\mathrm{O}$ que conclui a demonstração.

Em Souza et al. (2013) foi apresentada a seguinte lei de controle com realimentação do vetor de estado: 


$$
\begin{aligned}
u(t)=u_{\alpha}(t)=-\sum_{i=1}^{r} \alpha_{i} K_{i} x(t) & =-K(\alpha) x(t), \\
K_{i} & \in \mathbb{R}^{m \times n}, \quad i \in \mathbb{K}_{r} .
\end{aligned}
$$

Substituindo (13) em (7), obtém-se o sistema realimentado

$$
\begin{aligned}
\dot{x}(t)=A(\alpha) x(t) & -B(\alpha) K(\alpha) x(t) \\
& =\sum_{i=1}^{r} \sum_{j=1}^{r} \alpha_{i} \alpha_{j}\left(A_{i}-B_{i} K_{j}\right) x(t) .
\end{aligned}
$$

Foi justificado que a lei de controle (13) é fictícia, visto que $\alpha_{i}, i \in \mathbb{K}_{r}$, são parâmetros incertos que podem ser indisponíveis para medição, porém foi utilizada na análise do controlador chaveado que foi proposto.

Teorema 3. (Souza et al. (2013)). O ponto de equilíbrio $x=0$ do sistema linear com incertezas politópicas dado em (14) é globalmente assintoticamente estável, com taxa de decaimento maior ou igual $\gamma>0$, se existem uma matriz simétrica definida positiva $X(X \succ 0)$ e matrizes $M_{i} \in \mathbb{R}^{n \times m}$ tais que, para todo $i, j \in \mathbb{K}_{r}$, as seguintes LMIs sejam factíveis:

$$
\begin{aligned}
& X A_{i}^{T}+A_{i} X-B_{i} M_{i}-M_{i}^{T} B_{i}^{T}+2 \gamma X \prec 0, \\
& \quad\left(A_{i}+A_{j}\right) X+X\left(A_{i}+A_{j}\right)^{T}-B_{i} M_{j} \\
& \quad-B_{j} M_{i}-M_{i}^{T} B_{j}^{T}-M_{j}^{T} B_{i}^{T}+4 \gamma X \preceq 0, i<j .
\end{aligned}
$$

Se (15) e (16) são factíveis, os ganhos do controlador são dados por $K_{i}=M_{i} X^{-1}, i \in \mathbb{K}_{r}$.

Prova. Pode ser vista em Souza et al. (2013).

Teorema 4. (Souza et al. (2013)). Admita que as condições do Teorema 3 , relativas ao sistema (7) com a lei de controle (13), sejam satisfeitas e obtenha $K_{i}=M_{i} X^{-1}$, $i \in \mathbb{K}_{r}$, e $P=X^{-1}$. Então, a lei de controle chaveada proposta em Souza et al. (2013) torna o sistema (7) uniformemente "ultimate bounded".

Prova. Pode ser vista em Souza et al. (2013).

Observação 1. Neste trabalho não será exposta a lei de controle chaveada proposta em Souza et al. (2013), visto que não será projetado sistemas de controle com ela, apenas serão utilizadas as LMIs 15 e 16 para comparar região de factibilidade com o resultado proposto.

Resultados que estabelecem condições LMIs que garantem restrições na entrada e saída do sinal de controle podem ser vistos, por exemplo, em Boyd et al. (1994). Também, pode-se restringir a entrada do sinal de controle, de forma indireta, limitando a norma dos ganhos do controlador, como proposto em Siljak and Stipanović (2000), impondo restrições em $M_{i}, i \in \mathbb{K}_{r}$ e $X^{-1}$. Assim, dadas as constantes $\eta>0$ e $\eta_{x}>0$, impondo que $M_{i}^{T} M_{i} \prec \eta I_{n}, i \in \mathbb{K}_{r}$ e $X^{-1} \prec \eta_{x} I_{n}$, uma restrição sobre os ganhos do controlador pode ser estabelecida pelo teorema a seguir.

Teorema 5. (Šiljak and Stipanović (2000)). A restrição na norma dos ganhos do controlador tal que $K_{i} K_{i}^{T} \leq$ $\eta \eta_{x}^{2} I_{m}, i \in \mathbb{K}_{r}$, é imposta, se existem constantes $\eta>0$ e $\eta_{x}>0$, tais que as LMIs dos Teoremas 3 ou 4 (ou 1 ou 2 substituindo $K_{i}=K$ e $M_{i}=M$ ), com as LMIs abaixo, sejam factíveis.

$$
\left[\begin{array}{cc}
\eta_{x} I_{n} & I_{n} \\
I_{n} & X
\end{array}\right] \succeq 0, \quad\left[\begin{array}{cc}
\eta I_{n} & M_{i}^{T} \\
M_{i} & I_{m}
\end{array}\right] \succeq 0, \quad i \in \mathbb{K}_{r} .
$$

Prova. É análoga à apresentada em Šiljak and Stipanović (2000).

\section{RESULTADOS PRINCIPAIS}

Considere, agora, o sistema linear com incertezas politópicas:

$$
\dot{x}(t)=A(\alpha) x+B(\alpha) u_{\nu}
$$

Sendo $x \in \mathbb{R}^{n}, A(\alpha), B(\alpha) \in \mathcal{A}, A_{i} \in \mathbb{R}^{n \times n}, B_{i} \in \mathbb{R}^{n \times m}$, e $u_{\nu} \in \mathbb{R}^{m}$.

Define-se a lei de controle chaveada:

$$
u_{\nu}=-K_{\nu} x, \quad K_{\nu} \in \mathbb{R}^{m \times n}, \quad \nu=\arg \min _{i \in \mathbb{K}_{r}}\left\{x^{T} Q_{i} x\right\}
$$

A determinação do ganho do controlador é baseado em (Souza, 2013) e seleciona um ganho, que pertence a um conjunto de ganhos $\left\{K_{1}, K_{2}, \cdots, K_{r}\right\}$, por meio da seleção do índice $\nu=\arg \min _{i \in \mathbb{K}_{r}}\left\{x^{T} Q_{i} x\right\}$ sendo $\left\{Q_{1}, Q_{2}, \cdots, Q_{r}\right\}$ matrizes simétricas definidas positivas auxiliares.

Substituindo (19) em (18), tem-se:

$$
\dot{x}=A(\alpha) x-B(\alpha) K_{\nu} x=\left(A(\alpha)-B(\alpha) K_{\nu}\right) x
$$

E para garantir que o sistema linear realimentado (20) é estável estabelece-se o seguinte teorema.

Teorema 6. Suponha a existência de matrizes simétricas definidas positivas $X, R_{i} \in \mathbb{R}^{n \times n}$, matrizes simétricas $S_{i} \in \mathbb{R}^{n \times n}$, matrizes $M_{i} \in \mathbb{R}^{m \times n}$ e $\beta<0$ tais que, para todo $i, j \in \mathbb{k}_{r}$ :

$$
\begin{array}{r}
A_{i} X+X A_{i}^{T}-\beta X+S_{i}+R_{i} \prec 0 ; \\
-B_{i} M_{j}-M_{j}^{T} B_{i}^{T}-S_{i}-R_{j} \prec 0 .
\end{array}
$$

Então a lei de controle chaveada (19) torna o ponto de equilíbrio $x=0$ do sistema (18) globalmente assintoticamente estável, sendo $Q_{i}=X^{-1} R_{i} X^{-1}, \quad P=X^{-1}$ e os ganhos do controlador dados por $K_{j}=M_{j} X^{-1}, j \in \mathbb{k}_{r}$.

Prova. Considere uma candidata a função Lyapunov quadrática, $V=x^{T} P x$. Assim, $\dot{V}(x)=\dot{x}^{T} P x+x^{T} P \dot{x}$, de (20) segue que

$$
\begin{aligned}
& \dot{V}(x)-\beta V(x) \\
= & x^{T}\left(A(\alpha)-B(\alpha) K_{\nu}\right)^{T} P x+x^{T} P\left(A(\alpha)-B(\alpha) K_{\nu}\right) x \\
& \quad-\beta x^{T} P x \\
= & x^{T} A(\alpha)^{T} P x+x^{T} P A(\alpha) x-x^{T} \beta P x-x^{T} B(\alpha)^{T} K_{\nu}^{T} P x \\
& \quad-x^{T} P B(\alpha) K_{\nu} x \\
= & \sum_{i=1}^{r} \alpha_{i} x^{T}\left[A_{i}^{T} P+P A_{i}-\beta P-B_{i}^{T} K_{\nu}^{T} P-P B_{i} K_{\nu}\right] x
\end{aligned}
$$

Agora, suponha que existam matrizes simétricas $Z_{j}$ e $Q_{i}$, com $Q_{i} \succ 0$, tais que:

$$
-\left(P B_{i} K_{j}+K_{j}^{T} B_{i}^{T} P\right) \prec Z_{i}+Q_{j}, \forall i, j \in \mathbb{k}_{r}
$$

Portanto, de (23) e (24) segue que: 


$$
\begin{gathered}
\dot{V}(x)-\beta V(x) \leq \sum_{i=1}^{r} \alpha_{i} x^{T}\left[A_{i}^{T} P+P A_{i}-\beta P+Z_{i}+Q_{\nu}\right] x \\
\quad=\sum_{i=1}^{r} \alpha_{i} x^{T}\left[A_{i}^{T} P+P A_{i}-\beta P+Z_{i}\right] x+x^{T} Q_{\nu} x
\end{gathered}
$$

De (19), pode-se notar que

$$
x^{T} Q_{\nu} x=\min _{i \in \mathbb{k}_{r}}\left\{x^{T} Q_{i} x\right\} \leq \sum_{i=1}^{r} \alpha_{i} x^{T} Q_{i} x=x^{T} Q(\alpha) x .(26)
$$

Logo, substituindo (26) em (25) segue que

$$
\begin{aligned}
& \dot{V}(x)-\beta V(x) \\
\leq & \sum_{i=1}^{r} \alpha_{i} x^{T}\left[A_{i}^{T} P+P A_{i}-\beta P+Z_{i}\right]+\sum_{i=1}^{r} \alpha_{i}\left(x^{T} Q_{i} x\right) \\
\leq & \sum_{i=1}^{r} \alpha_{i} x^{T}\left[A_{i}^{T} P+P A_{i}-\beta P+Z_{i}+Q_{i}\right] x
\end{aligned}
$$

Lembrando que $\alpha_{i}>0, i \in \mathbb{k}_{r}$, e $\sum_{i=1}^{r} \alpha_{i}=1 . \dot{V}(x)<0$ (para todo $x \neq 0$ ), se

$$
A_{i}^{T} P+P A_{i}-\beta P+Z_{i}+Q_{i} \prec 0 .
$$

Multiplicando (24) e (28) por $P^{-1}$ de ambos os lados obtém-se

$$
\begin{gathered}
-B_{i} K_{j} P^{-1}-P^{-1} K_{j}^{T} B_{i}^{T}-P^{-1} Z_{i} P^{-1}-P^{-1} Q_{j} P^{-1} \prec 0 ;(29) \\
P^{-1} A_{i}^{T}+A_{i} P^{-1}-\beta P^{-1}+P^{-1} Z_{i} P^{-1}+P^{-1} Q_{i} P^{-1} \prec 0 .(30)
\end{gathered}
$$

Agora, defina $X=P^{-1}, M_{j}=K_{j} X, S_{i}=P^{-1} Z_{i} P^{-1} \mathrm{e}$ $R_{j}=P^{-1} Q_{j} P^{-1}$. Portanto, reescrevendo as equações (30) e (29) com as devidas substituições segue que

$$
\begin{gathered}
-B_{i} M_{j}-M_{j}^{T} B_{i}^{T}-S_{i}-R_{j} \prec 0 ; \\
X A_{i}^{T}+A_{i} X-\beta X+S_{i}+R_{i} \prec 0 .
\end{gathered}
$$

Concluindo a demonstração.

\section{EXEMPLOS}

Nesta seção, será utilizado um exemplo para ilustrar que as LMIs estabelecidas para encontrar os ganhos do controlador, propostas no Teorema 6 , são menos conservadoras do que as LMIs clássicas (Teoremas 2), entretanto, são iguais às propostas em Souza et al. (2013) (Teoremas 3 e 4).

Em Souza et al. (2013) foi considerado o sistema linear incerto dado em (7), sendo

$$
x(t)=\left[\begin{array}{l}
x_{1} \\
x_{2}
\end{array}\right], \quad A(\alpha)=\left[\begin{array}{cc}
0 & 1 \\
\bar{a} & -1
\end{array}\right] \quad \text { e } \quad B(\alpha)=\left[\begin{array}{c}
0 \\
\bar{b}
\end{array}\right],
$$

com $-27 \leq \bar{a} \leq a,-10 \leq \bar{b} \leq b,-20 \leq a \leq 15 \mathrm{e}$ $-9 \leq b \leq-1$.

Portanto, os vértices do politopo são como seguem:

$$
\begin{gathered}
A_{1}=A_{2}=\left[\begin{array}{cc}
0 & 1 \\
a & -1
\end{array}\right], A_{3}=A_{4}=\left[\begin{array}{cc}
0 & 1 \\
-27 & -1
\end{array}\right], \\
B_{1}=B_{3}=\left[\begin{array}{l}
0 \\
b
\end{array}\right], B_{2}=B_{4}=\left[\begin{array}{c}
0 \\
-10
\end{array}\right] .
\end{gathered}
$$

Como em Souza et al. (2013), serão encontradas as regiões de factibilidade do sistema e, para assegurar uma comparação justa, foi especificado no Teorema 3 uma taxa de decaimento maior ou igual a $1,5(\gamma=3)$, no Teorema 6 , $\beta=-6$, e no Teorema 5 , para a restrição da norma dos ganhos do controlador, foram fixados $\eta=400$ e $\eta_{x}=2$. Assim, da Figura 1 observa-se que o método proposto tem uma região factível igual a obtida com o Teorema 3.



Figura 1. Regiões factíveis utilizando os Teoremas 3 e 5 $("+")$ e o método proposto, Teoremas 6 e 5 ("०").

Como pode ser observado na Figura 1, o método proposto tem uma região de factibilidade igual a obtida com o Teorema 3. Porém, não há necessidade de adicionar o integrador, mantendo, portanto, as dimensões originais das matrizes.

\subsection{O Levitador Magnético}

A aplicação proposta para o artigo é em um levitador magnético, que possui a sua forma de movimentação mostrada na Figura 2, Cardim (2009). O levitador magnético compreende de um eletroímã, obtido através de uma bobina que envolve o núcleo de material ferromagnético, dessa forma, o campo magnético atua sobre uma bola, mantendo-a sobre seu efeito sem contato físico com o eletroímã.

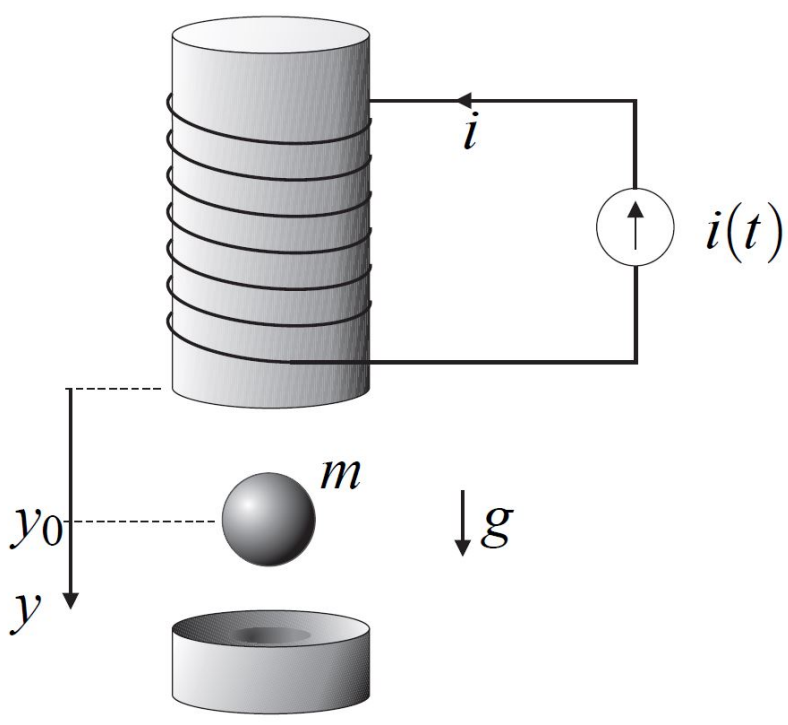

Figura 2. modelo esquemático de um levitador magnético. 
O modelo matemático do levitador magnético, ilustrado na Figura 2, (Marquez, 2003, p. 24), é dado por

$$
m \ddot{y}=-k \dot{y}+m g-\frac{\lambda \mu i^{2}}{2(1+\mu y)^{2}},
$$

sendo os valores da constantes dados na Tabela 1 .

Tabela 1. Valores das constantes

\begin{tabular}{lcc} 
Constantes & Parâmetros & Valor Numérico \\
\hline Massa da Bola (incerta) & $\mathrm{m}$ & $--g$ \\
Aceleração da Gravidade & $\mathrm{g}$ & $9,8 \frac{\mathrm{m}}{\mathrm{s}^{2}}$ \\
Permeabilidade magnética & $\mu$ & $2 \mathrm{~m}^{-1}$ \\
Parâmetro (incerto) & $\lambda$ & $--[H]$ \\
Parâmetro constante & $k$ & $0,001 \mathrm{Ns} / \mathrm{m}$ \\
Corrente & $i$ & \\
Posição da bola & $y$ & \\
\hline
\end{tabular}

Como em Cardim (2009), definindo as variáveis de estado $\bar{x}_{1}=y$ e $\bar{x}_{2}=\dot{y}$ e como entrada $i$, a equação (31) pode ser escrita como segue:

$$
\left\{\begin{array}{l}
\dot{\bar{x}}_{1}=\bar{x}_{2}, \\
\dot{\bar{x}}_{2}=g-\frac{k}{m} \bar{x}_{2}-\frac{\lambda \mu i^{2}}{2 m\left(1+\mu \bar{x}_{1}\right)^{2}} .
\end{array}\right.
$$

Considerou-se que durante a operação requerida $\left[\begin{array}{ll}\bar{x}_{1} & \bar{x}_{2}\end{array}\right]^{T} \in$ $D$, sendo

$$
\begin{aligned}
D=\left\{\left[\begin{array}{ll}
\bar{x}_{1} \quad \bar{x}_{2}
\end{array}\right]^{T} \in \mathbb{R}^{2}: 0 \leq \bar{x}_{1} \leq 0,15\right. \\
0,03 \leq m \leq 0,09, \quad 0,26 \leq \lambda \leq 0,66\} .
\end{aligned}
$$

O objetivo deste exemplo é projetar um controlador que mantenha a bola em uma posição desejada $y=\bar{x}_{1}=y_{0}$. Assim, o ponto de equilíbrio do sistema (32) é $\bar{x}_{e}=$ $\left[\begin{array}{ll}\bar{x}_{1_{e}} & \bar{x}_{2_{e}}\end{array}\right]^{T}=\left[\begin{array}{ll}y_{0} & 0\end{array}\right]^{T}$.

Da segunda equação de (32), observou-se que no ponto de equilíbrio, $\dot{\bar{x}}_{2}=0$ e $i=i_{0}$, sendo $i_{0}=\sqrt{\frac{2 m g}{\lambda \mu}\left(1+\mu y_{0}\right)^{2}}$.

Linearizando o sistema (32) em torno do ponto de equilíbrio $\bar{x}=\bar{x}_{e}$ e $i=i_{0}$ obteve-se

$$
\left[\begin{array}{c}
\dot{x}_{1} \\
\dot{x}_{2}
\end{array}\right]=\left[\begin{array}{cc}
0 & 1 \\
\frac{2 g \mu}{1+\mu y_{0}} & -\frac{k}{m}
\end{array}\right]\left[\begin{array}{l}
x_{1} \\
x_{2}
\end{array}\right]+\left[\begin{array}{c}
0 \\
\frac{-\sqrt{2 \lambda \mu g}}{\sqrt{m}\left(1+\mu y_{0}\right)}
\end{array}\right] u
$$

sendo

$$
\left\{\begin{array} { l } 
{ x _ { 1 } = \overline { x } _ { 1 } - y _ { 0 } , } \\
{ x _ { 2 } = \overline { x } _ { 2 } , } \\
{ u = i - i _ { 0 } , }
\end{array} \Rightarrow \left\{\begin{array}{l}
\bar{x}_{1}=x_{1}+y_{0} \\
\bar{x}_{2}=x_{2} \\
i=u+i_{0}
\end{array}\right.\right.
$$

ou seja,

$$
\left\{\begin{array}{l}
\dot{\bar{x}}_{1}=\dot{x}_{1} \\
\dot{\bar{x}}_{2}=\dot{x}_{2} \\
i=u+\sqrt{\frac{2 m g}{\lambda \mu}\left(1+\mu y_{0}\right)^{2}} .
\end{array}\right.
$$

Nota-se que a equação (34) está na forma apresentada em (7), considerando:

$$
A=\left[\begin{array}{cc}
0 & 1 \\
\frac{2 g \mu}{1+\mu y_{0}} & -\frac{k}{m}
\end{array}\right] \quad \text { e } B=\left[\begin{array}{c}
0 \\
\frac{-\sqrt{2 \lambda \mu g}}{\sqrt{m}\left(1+\mu y_{0}\right)}
\end{array}\right] .
$$

\subsection{Simulações Numéricas}

Para a simulação foi considerado que a massa $m$ e o parâmetro $\lambda$ são incertos com seus valores variando conforme estabelecidos no conjunto $D$, dado em (33). A incerteza no parâmetro $\lambda$ simula uma interferência que faz variar a indutância, simulando uma falha nas características construtivas do eletroímã. O processo produz um politopo de quatro vértices, cujas incertezas politópicas estão inserida nas matrizes $A$ e $B$. Assim, tem-se os seguintes vértices do politopo

$$
\begin{gathered}
A_{1}=A_{2}=\left[\begin{array}{cc}
0 & 1 \\
31,1111 & -0,0333
\end{array}\right] \\
A_{3}=A_{4}=\left[\begin{array}{cc}
0 & 1 \\
31,1111 & -0,0111
\end{array}\right] \\
B_{1}=B_{3}=\left[\begin{array}{c}
0 \\
-23,3069
\end{array}\right], \quad B_{2}=B_{4}=\left[\begin{array}{c}
0 \\
-8.4457
\end{array}\right] .
\end{gathered}
$$

Utilizando o MATLAB, com a linguagem computacional do YALMIP e o solver SeDuMi, considerando os vértices do politopo (35) e $\beta=0$, foram encontradas as matrizes simétricas definidas positivas $P, Q_{1}, Q_{2}, Q_{3}$ e $Q_{4}$ e os ganhos do controlador $K_{1}, K_{2}, K_{3}$ e $K_{4}$ utilizado o teorema proposto (Teorema 6 )

$$
P=\left[\begin{array}{cc}
102,5891 & 18,2366 \\
18,2366 & 4,1632
\end{array}\right]
$$

$Q_{1}=10^{4}\left[\begin{array}{ll}1,3462 & 0,2432 \\ 0,2432 & 0,0440\end{array}\right], Q_{2}=10^{4}\left[\begin{array}{ccc}1,0342 & 0,1814 \\ 0,1814 & 0,0320\end{array}\right]$,

$Q_{3}=10^{4}\left[\begin{array}{l}1,34710,2434 \\ 0,24340,0440\end{array}\right], Q_{4}=10^{4}\left[\begin{array}{ll}1,0299 & 0,1805 \\ 0,1805 & 0,0318\end{array}\right]$,

$K_{1}=[-6,9400-1,0316], K_{2}=[-11,0439-1,7408]$,

$K_{3}=[-6,9269-1,0296], K_{4}=[-11,1486-1,7596]$,

e considerando o método clássico (Teorema 2) a seguinte matriz definida positiva e o ganho, foram obtidos:

$$
\begin{gathered}
P=\left[\begin{array}{cc}
0,0136 & -0.0689 \\
-0,06890,4680 &
\end{array}\right], \\
K=[-10,3541-1,3855]
\end{gathered}
$$

Para a simulação numérica foi considerada a condição inicial $\bar{x}(0)=\left[\begin{array}{ll}0,02 & 0\end{array}\right]^{T}, m=0,08 \mathrm{~g}$ e $\lambda=0,5 \mathrm{H}$ e a posição de equilíbrio $y_{0}=0,13 \mathrm{~m}$, obtendo, portanto, $i_{0}=1,5778$ que é a corrente necessária para manter a bola flutuando. Os resultados obtidos são mostrados na Figura 3.

Como pode ser visto na Figura 3, o novo projeto de controlador apresentou um bom desempenho, pois apresentou um tempo de estabelecimento adequado e exigiu pouco esforço de corrente para estabilizar a bola na posição desejada $y_{0}=0,13 \mathrm{~m}$, confirmando a eficiência do método.

\section{CONCLUSÃO}

Neste trabalho, que é uma pesquisa básica, fruto de um trabalho de iniciação cientifica, foi proposta uma nova 




Figura 3. Posição $y(t)=\bar{x}_{1}(t)$, velocidade $\bar{x}_{2}(t)$, corrente elétrica $i(t)$ e chaveamento dos ganhos do controlador, do sistema controlado, utilizando o controlador chaveado (19) (linha contínua) e controlador clássico (3) (linha pontilhada), considerando a condição inicial $\bar{x}(0)=\left[\begin{array}{ll}0,02 & 0\end{array}\right]^{T}, y_{0}=0,13 \mathrm{~m}, m=0,08 \mathrm{~g}, \lambda=0.5 \mathrm{H}$ e $\beta=0$.

metodologia de sistema de controle chaveado para sistemas lineares com incertezas politópicas. A metodologia generaliza Souza et al. (2013) ao não exigir que a matriz $B$, do sistema (7), seja constante. Além disso, como pode ser visto na Figura 1, em termos de conservadorismo, as LMIs propostas são iguais às estabelecidas em Souza et al. (2013), que por sua vez são mais relaxadas do que as clássicas propostas em (Boyd et al., 1994), e na Figura 3 , mostra que a resposta do sistema controlado (31)-(19) apresentou um desempenho apropriado.

Como prosseguimento deste trabalho, pretende-se estabelecer, seguindo esta mesma linha de projeto de controle, critérios de desempenho como, por exemplo, $\mathcal{H}_{2}$ e $\mathcal{H}_{\infty}$.

\section{AGRADECIMENTOS}

Os autores agradecem ao $\mathrm{CNPq}$ e ao IFTO pelo apoio na pesquisa.

\section{REFERÊNCIAS}

Boyd, S., Ghaoui, L.E., Feron, E., and Balakrishnan, V. (1994). Linear matrix inequalities in system and control theory, volume 15 of SIAM Studies in Applied Mathematics. SIAM Society for Industrial and Applied Mathematics, Philadelphia, 2 edition.

Cardim, R. (2009). Projeto de Controladores Baseados em LMIs: realimentação derivativa e sistemas chaveados utilizando estrutura variável. Doutorado em engenharia elétrica, Ilha Solteira.

de Oliveira, D.R., Teixeira, M.C.M., Assunção, E., Souza, W.A., Moreira, M.R., and ao Henrique Pereira Silva, J. (2014). Projeto de controle robusto $\mathcal{H}_{\infty}$ chaveado: Implementação prática em um sistema de suspensão ativa. volume 2014, 8. Belo Horizonte.

Decarlo, R.A., Branicky, M.S., Pettersson, S., and Lennartson, B. (2000). Perspectives and results on the stability and stabilizability of hybrid systems. Proceedings of the IEEE, 88(7), 1069-1082.

Geromel, J.C. and Colaneri, P. (2006). Stability and stabilization of continuous-time switched linear systems. SIAM Journal on Control and Optimization, 45(5), 1915-1930.

Hespanha, J.P. and Morse, A.S. (2002). Switching between stabilizing controllers. Automatica, 38(11), 1905-1917.
Leite, V.J.S., de Oliveira, P.J., Ramos, D.C.W., Montagner, V.F., Oliveira, R.C.L.F., and Peres, P.L.D. (2004). Estabilidade robusta de sistemas lineares através de desigualdades matriciais lineares. Controle e Automação, $15(1), 24-40$.

Liberzon, D. and Morse, A.S. (1999). Basic problems in stability and design of switched systems. IEEE Control Systems, 19(5), 59-70.

Lofberg, J. (2004). YALMIP: a toolbox for modeling and optimization in MATLAB. In Proceedings..., 2004, 284-289. IEEE International Symposium on Computer Aided Control Systems Design, IEEE, Taipei.

Marquez, H.J. (2003). Nonlinear Control Systems: analysis and design. John Wiley Sons, Inc., New Jersey.

Šiljak, D.D. and Stipanović, D.M. (2000). Robust stabilization of nonlinear systems: the LMI approach. Mathematical Problems in Engineering, 6(5), 461-493.

Silva, J.H.P., Júnior, E.M., de Souza, W.A., Teixeira, M.C.M., Assunção, E., Cardim, R., and Moreira, M.R. (2012). Controle $\mathcal{H}_{\infty}$ com chaveamento do ganho da realimentação do vetor de estado para sistemas lineares incertos. In Anais ... Congresso Brasileiro de Automática - CBA, Campina Grande.

Souza, W.A., Oliveira, D.R.D., Teixeira, M.C.M., Silva, L.S.C., Cardim, R., and Assunção, E. (2014). Projeto e implementação de um controlador robusto chaveado utilizando modelos fuzzy takagi-sugeno. volume 2014, 8. Belo Horizonte.

Souza, W.A., Teixeira, M.C.M., Santim, M.P.A., Cardim, R., and Assunção, E. (2013). On switched control design of linear time-invariant systems with polytopic uncertainties. Mathematical Problems in Engineering, 2013, 10 .

Sturm, J.F. (1999). Using SeDuMi 1.02, a MATLAB toolbox for optimization over symmetric cones. Optimization Methods and Software, 11-12, 625-653.

Wicks, M.A., Peleties, P., and DeCarlo, R.A. (1994). Construction of piecewise Lyapunov functions for stabilizing switched systems. In Proceedings..., volume 4, 34923497. Conference on Decision and Control, IEEE, Lake Buena Vista. 